Pacific Journal of Mathematics

BOUNDS FOR THE NUMBER OF DEFICIENT VALUES O
ENTIRE FUNCTIONS WHOSE ZEROS HAVE ANGULAR

KI-Choul OUM 


\title{
BOUNDS FOR THE NUMBER OF DEFICIENT VALUES OF ENTIRE FUNCTIONS WHOSE ZEROS HAVE ANGULAR DENSITIES
}

\author{
KI-CHOUL OUM
}

Let $f(z)$ be an entire function of finite order $\lambda$. Arakeljan has shown that, for every $\lambda>1 / 2, f(z)$ may have infinitely many deficient values in the sense of $R$. Nevanlinna. We prove here that this cannot happen if (i) the zeros of $f(z)$ have an angular density (in the sense of Pfluger and Levin) and (ii) $\lambda$ is not an integer. Under these two assumptions the number of deficient values cannot exceed $2 \lambda+1$. If $f(z)$ is of completely regular growth (in the sense of Levin), the result also holds for integral values of the order.

We use systematically the fundamental concepts of Nevanlinna's theory of meromorphic functions and take for granted the meaning of the following well established symbols of this theory

$$
\log ^{+}, M(r, f), m(r, f), n(r, \tau), N(r, \tau), T(r, f) \text {. }
$$

Since $n(r, o)$ is the only one of the functions $n(r, \tau)$ which concerns us, we set $n(r)=n(r, o)$, and use the more detailed notation

$$
n\left(r ; \phi_{1}, \phi_{2}\right) \quad\left(o<\phi_{2}-\phi_{1} \leqq 2 \pi\right),
$$

to indicate the number of zeros of $f(z)$ lying in the sector

$$
\left\{z:|z| \leqq r, \phi_{1} \leqq \phi<\phi_{2}\right\} \quad(\phi=\arg z) .
$$

Multiple zeros are counted in (1) as often as indicated by their multiplicities.

The deficiency $\delta(\tau, f)$ of the value $\tau$ (of the function $f$ ) is defined by

$$
\delta(\tau, f)=1-\limsup _{r \rightarrow \infty} \frac{N(r, \tau)}{T(r, f)} .
$$

I confine my study to entire functions of finite order $\lambda$ :

$$
\lambda=\limsup _{r \rightarrow+\infty} \frac{\log \log M(r, f)}{\log r},
$$

and always denote by $\nu(f)(\leqq+\infty)$ the number of distinct values of $\tau$ for which $\delta(\tau, f)>0$. Since $f(z)$ is entire, $\delta(\infty, f)=1$ and hence

$$
1 \leqq \nu(f) \leqq+\infty \text {. }
$$


Before the important discovery of Arakeljan [1], it had been conjectured, for a long time, that, in the case of entire functions, $\lambda<+\infty$ implies $\nu(f)<+\infty$.

The erroneous conjecture was based on the intuitive idea that the link between the notions of deficient and asymptotic values is strong enough to imply

$$
\nu(f) \leqq 2 \lambda+1
$$

(in complete analogy with Ahlfors' sharp bound for the number of asymptotic values, including $\infty$, of an entire function of order $\lambda$ ).

The main result of the present paper may be stated as

THEOREM 1. Let $f(z)$ be an entire function of finite nonintegral order $\lambda$, and let the zeros of $f(z)$ have an angular density in the sense of Pfluger and Levin.

Then the number of deficient values of $f(z)$ is necessarily finite and cannot exceed $2 \lambda+1$. If $2 \lambda$ is a positive integer, the number of deficient values cannot exceed $2 \lambda$.

The notion of angular density, which appears in the above statement, requires the preliminary introduction of a positive, continuous, nondecreasing function $V(r)$ whose growth is: tions;

(i) regular enough to lead to manageable asymptotic computa-

(ii) so "close" to the growth of $\log M(r, f)$ that

$$
\limsup _{r \rightarrow \infty} \frac{\log M(r, f)}{V(r)}=1 \text {. }
$$

Following a well established pattern, we shall always take

$$
V(r)=r^{\lambda(r)},
$$

where $\lambda(r)$ is any one of the Lindelöf proximate orders of $f(z)$.

We say that the function $V(r)=V(r, f)$ thus chosen is a Valiron growth function of $f(z)$.

The difinition of $\lambda(r)$ (and hence also the definition of $V(r)$ ), its existence and elementary properties are well-known and will be taken for granted. Proofs and a detailed account of the relevant results will be found in [2; pp. $54 \sim 58]$.

Following Pfluger [7;p.204], we say that the zeros of $f(z)$ have an angular density, with respect to $V(r)=V(r, f)$, if, with every $\varepsilon>0$, it is possible to associate a finite number $k=k(\varepsilon)$ of arguments:

$$
\phi_{1}, \phi_{2}, \cdots, \phi_{k} \quad\left(0 \leqq \phi_{1}<\phi_{2}<\cdots<\phi_{k}<2 \pi\right)
$$


such that

$$
\phi_{j+1}-\phi_{j}<\varepsilon \quad\left(j=1,2, \cdots, k ; \phi_{k+1}=\phi_{1}+2 \pi\right),
$$

and such that the limits

$$
\lim _{r \rightarrow \infty} \frac{n\left(r ; \phi_{j}, \phi_{j+1}\right)}{r^{2(n)}}
$$

exist (and are finite) for $j=1,2, \cdots, k$.

In Theorem 1 we assume that "the zeros of $f(z)$ have an angular density" and make no reference to the corresponding $V(r)$. This is to be understood as follows: the zeros of $f(z)$ have an angular density with respect to some Valiron growth function of $f(z)$.

We conclude this Introduction by pointing out that Edrei and Fuchs [3] proved the inequality

$$
\nu(f) \leqq 2 \lambda+2
$$

for entire functions of order $\lambda$ satisfying conditions quite different from the ones considered here. Their remarkable Theorems 3 and 4, in conjunction with our Theorems 1 and 2, show that, although the bound in (7) is not general, it is very likely to hold for all the entire functions, arising "naturally" in the course of an analytical investigation.

1. Functions of completely regular growth; statement of Theorem 2. The indicator function $h(\phi)$, of Phragmén-Leindelöf, plays an important role in our proofs. We define

$$
h(\dot{\phi})=\limsup _{r \rightarrow+\infty} \frac{\log \left|f\left(r e^{i \phi}\right)\right|}{V(r)},
$$

and remind the reader that, in view of (5) and $(6), h(\phi)$ is a continuous function; it is always finite, does not vanish identically, and satisfies the fundamental inequality [2; pp. $41 \sim 46$ and p. 54]

(1.2) $h\left(\dot{\phi}_{1}\right) \sin \lambda\left(\dot{\phi}_{3}-\dot{\phi}_{2}\right)+h\left(\dot{\phi}_{2}\right) \sin \lambda\left(\dot{\phi}_{1}-\dot{\phi}_{3}\right)+h\left(\dot{\phi}_{3}\right) \sin \lambda\left(\dot{\phi}_{2}-\phi_{1}\right) \geqq 0$,

valid for

$$
\dot{\phi}_{1}<\dot{\phi}_{2}<\dot{\phi}_{3}, \dot{\phi}_{2}-\dot{\phi}_{1}<\frac{\pi}{\lambda}, \dot{\phi}_{3}-\dot{\phi}_{2}<\frac{\pi}{\lambda} ; \lambda=\lim _{r \rightarrow \infty} \lambda(r) .
$$

It will be noticed that Theorem 1 makes no assertions concerning functions of integral order. This is due to the fact that, if $\lambda$ is an integer, the connections between the zeros of $f(z)$ and its indicator $h(\phi)$ are not simple. In all other respects our proof of Theorem 1 requires no modifications when $\lambda$ is a positive integer. This leads us 
to adopt the notion of function of completely regular growth systematically investigated by Levin [5; Chap. III], and to prove [Theorem 2] a generalization of Theorem 1 .

Definition of completely regular growth. Put

$$
h(r, \phi)=\frac{\log \left|f\left(r e^{i \phi}\right)\right|}{V(r)}
$$

and let $\mathscr{E}$ be some set of values of $r$, of density zero. Following Levin, we say that $f(z)$ is of completely regular growth, with respect to $V(r)$, if the following convergence

$$
h(r, \phi) \rightarrow h(\phi)
$$

takes place, uniformly in $\phi$, as $r \rightarrow+\infty$ and avoids some possible exceptional set $\mathscr{E}$, of density zero.

For sake of completeness we remind the reader that a set $\mathscr{E}$ is of density zero if (i) it is measurable and (ii) the portion $\mathscr{E}_{r}$, of $\mathscr{E}$, which falls in the interval $[0, r]$ satisfies the condition

$$
\lim _{r \rightarrow \infty} \frac{\text { meas } \mathscr{E}_{r}}{r}=0 \text {. }
$$

From now on, we shall always denote by $\mathscr{E}$ the exceptional set (of values of $r$ ) which appears in the definition of completely regular growth, and by $C$ the set of all arguments $\phi$ :

$$
0 \leqq \phi \leqq 2 \pi \text {. }
$$

Since we are only interested in the circular arrangement of the elements of $C$, the points $\phi=0$ and $\phi=2 \pi$ will be "identified" and, more generally, all the values

$$
\phi+2 k \pi \quad(k=0, \pm 1, \pm 2, \cdots)
$$

will be considered as different numerical representation of a single element of $C$.

Consider the subset of $C$ defined by

$$
C_{0}=\{\phi: h(\phi)=0\},
$$

where $h(\phi)$ is the indicator of an entire function of finite order $\lambda$, and of completely regular growth.

In $\S 7$ we shall prove

LEMma 1. The set $C_{0}$ is the union of finitely many isolated 
points and of $q$ disjoint closed intervals which do not reduce to points:

$$
0 \leqq q \leqq 2 \lambda
$$

Lemma 1 is stated at this early stage because it clarifies the assertions of our most general result:

THEOREM 2. Let $f(z)$ be an entire function of order $\lambda$,

$$
0<\lambda<+\infty \text {. }
$$

Assume that $f(z)$ is of completely regular growth with respect to one of its Valiron growth functions and let $q$ be the number of component intervals, which do not reduce to points, of the set $C_{0}$.

Then the number of deficient values of $f(z)$, other than 0 and $\infty$, cannot exceed $q$ and

$$
\nu(f) \leqq 2 \lambda+1
$$

If $2 \lambda$ is a positive integer, (1.9) is to be replaced by the sharper inequality

$$
\nu(f) \leqq 2 \lambda
$$

In the case of functions of finite, nonintegral order, the existence of an angular density of zeros implies complete regularity of the growth. (This was shown by Pfluger [7; p. 206, Th. 3] and Levin [5; p. 90].) Hence Theorem 1 follows immediately from Theorem 2.

We finally remark that the well known function $[6 ;$ p. 170]

$$
f(z)=\int_{0}^{z} \exp \left(-t^{q}\right) d t \quad(q \geqq 2),
$$

of order $q$, is of completely regular growth and shows that the first assertion of Theorem 2 is "best possible". The inequalities (1.9) and (1.10) are perhaps less satisfactory and all the examples known to the author do not preclude the sharper form

$$
\nu(f) \leqq \lambda+1 \text {. }
$$

2. Notational conventions and statement of known results. Closed intervals such as $a \leqq x \leqq b(a \leqq b)$ are denoted by $[a, b]$. All the sets which appear in this note are measurable. Following Edrei and Fuchs, we define

$$
m(r, f ; J)=\frac{1}{2 \pi} \int_{J} \log ^{+}\left|f\left(r e^{i \phi}\right)\right| d \phi,
$$


where $J(\subset C)$ is measurable.

It is convenient to introduce systematically

$$
\begin{gathered}
h^{+}(\phi)=\left\{\begin{aligned}
h(\phi) & \text { if } h(\phi) \geqq 0, \\
0 & \text { if } h(\phi)<0,
\end{aligned}\right. \\
H=\frac{1}{2 \pi} \int_{0}^{2 \pi} h(\phi) d \phi, \quad H_{+}=\frac{1}{2 \pi} \int_{0}^{2 \pi} h^{+}(\phi) d \dot{\phi} .
\end{gathered}
$$

We denote positive constants depending on one or several parameters by $K$. Most of our inequalities are only valid for sufficiently large values of $r$. We indicate this fact by writing, immediately after the relevant inequality, $\left(r>r_{0}\right)$. The quantities $K$ and $r_{0}$ are not necessarily the same ones each time they occur.

For the convenience of the reader we state two lemmas of Edrei and Fuchs which are of particular importance in our proofs.

Lemma A [4; p. 322, Lemma III]. Let $g(z)$ be meromorphic. With each $r(>0)$ we associate a set $\Lambda(r)$ (of values of $\phi$ ) such that

$$
\text { meas } \Lambda(r)=\mu(r) \text {. }
$$

Then, for $1<r<R^{\prime}$, we have

$$
m(r, g ; \Lambda(r)) \leqq \frac{11 R^{\prime}}{R^{\prime}-r} T\left(R^{\prime}, g\right) \mu(r)\left[1+\log ^{+} \frac{1}{\mu(r)}\right] .
$$

Lemma B $[4 ;$ p. 321, Lemma I]. Let $W(z)$ be regular, except for poles

$$
c_{1}, c_{2}, \cdots, c_{l},
$$

in the sector

$$
s: \frac{r}{\sigma} \leqq|z| \leqq \sigma r,|\arg z-\xi| \leqq \gamma,
$$

where $\xi$ is real and

$$
r>0, \sigma>1,0<\gamma \leqq \pi, 2 \log \sigma \leqq \pi \gamma .
$$

Let

$$
|W(z)| \leqq 1
$$

on the boundary of $S$. Write $J$ for the intervals of $\phi^{\prime} s$

$$
J:|\dot{\phi}-\xi| \leqq(1-2 \delta) \gamma \quad\left(0<\delta<\frac{1}{4}\right) .
$$

Then, for $\phi \varepsilon J$, 


$$
\begin{aligned}
\log \left|W\left(r e^{i \phi}\right)\right| \leqq & -\frac{64 \delta^{6}}{\log \sigma} \exp \left(-16 \frac{\pi \gamma}{\log \sigma}\right) m\left(r, \frac{1}{W} ; J\right) \\
& +\log \left|\frac{(2 r \sigma)^{l}}{Q\left(r e^{i \phi}\right)}\right|,
\end{aligned}
$$

where

$$
Q(z)=\prod_{j=1}^{l}\left(z-c_{j}\right)
$$

(if there are no poles of $W(z)$ in $S$, then the last term on the righthand side of (2.3) is to be omitted).

3. Evaluation of $\delta(0, f)$ for functions of completely regular growth. We first prove an elementary lemma which shows that it is frequently possible to eliminate exceptional sets of density zero.

LEMma 2. Let $\chi(r)$ and $V(r)$ be positive, nondecreasing functions of $r\left(r>r_{0} \geqq 0\right)$ and let

$$
\lim _{r \rightarrow \infty} \frac{V(\sigma r)}{V(r)}=\sigma^{\lambda} \quad(0<\lambda<+\infty)
$$

for every fixed $\sigma>1$.

Denote by $\mathscr{E}_{0}$ a possible exceptional set of density zero. Then

$$
\lim _{\substack{r \rightarrow \infty \\ r \notin \notin 0}} \frac{\chi(r)}{V(r)}=B \quad(0 \leqq B<+\infty)
$$

implies

$$
\lim _{r \rightarrow \infty} \frac{\chi(r)}{V(r)}=B
$$

Proof. Let $\sigma>1$ be given. The fact that $\mathscr{E}_{0}$ has density zero implies the following: with each $r\left(r>r_{0}\right)$ it is possible to associate two values $r_{1}=r_{1}(r)$ and $r_{2}=r_{2}(r)$ such that

$$
r_{1} \notin \mathscr{E}_{0}, \quad r_{2} \notin \mathscr{E}_{0},
$$

and

$$
\frac{r}{\sigma}<r_{1}<r<r_{2}<\sigma r .
$$

Since $\chi(r)$ and $V(r)$ are nondecreasing functions of $r\left(>r_{0}\right)$,

$$
\frac{\chi\left(r_{1}\right)}{V\left(r_{1}\right)} \frac{V(r / \sigma)}{V(r)} \leqq \frac{\chi(r)}{V(r)} \leqq \frac{\chi\left(r_{2}\right)}{V\left(r_{2}\right)} \frac{V(\sigma r)}{V(r)} \quad\left(r>r_{0}\right) .
$$


Let $r \rightarrow \infty$ in the latter inequalities; then, by (3.5), $r_{1} \rightarrow \infty$ and $r_{2} \rightarrow \infty$. In view of (3.1), (3.2) and (3.4), we find

$$
B \sigma^{-\lambda} \leqq \liminf _{r \rightarrow \infty} \frac{\chi(r)}{V(r)} \leqq \limsup _{r \rightarrow \infty} \frac{\chi(r)}{V(r)} \leqq B \sigma^{\lambda} .
$$

Letting $\sigma \rightarrow 1+$, we obtain (3.3).

LEMma 3. Let $f(z)$ be an entire function of finite order and of completely regular growth with respect to one of its Valiron growth functions $V(r)$.

Then (with the notation introduced in (2.1)),

$$
\begin{aligned}
& 0<H_{+}<+\infty, \\
& T(r, f) \sim H_{+} V(r) \quad(r \rightarrow+\infty),
\end{aligned}
$$

and

$$
\delta(0, f)=\frac{H_{+}-H}{H_{+}} .
$$

Proof. The function $h(\phi)$ is continuous on $C$ (this well known property of the indicator holds in view of the conditions (5) and (6)). Hence there exists $\gamma \in C$ such that

$$
h(\gamma)=\sup _{\phi \in C} h(\dot{\phi}),
$$

and, by the uniformity of the convergence in (1.5),

$$
\log \left|f\left(r e^{i \gamma}\right)\right| \leqq \log M(r, f) \leqq(h(\gamma)+\epsilon) V(r) \quad\left(r>r_{0}, r \notin \mathscr{E}\right) .
$$

This implies

$$
\lim _{\substack{r \rightarrow \infty \\ r \notin \&}} \frac{\log M(r, f)}{V(r)}=h(\gamma),
$$

and, by (5) and Lemma 2,

$$
\lim _{r \rightarrow \infty} \frac{\log M(r, f)}{V(r)}=1=h(\gamma)=h^{+}(\gamma),
$$

The inequalities (3.6) follow from the continuity of $h^{+}(\phi),(2.1)$ and (3.9).

From (1.5) we deduce that

$$
|h(r, \phi)| \longrightarrow|h(\phi)| \quad(r \notin \mathscr{E}, r \longrightarrow+\infty),
$$

uniformly in $\phi$. Adding (1.5) and (3.10), we obtain 


$$
\frac{\log ^{+}\left|f\left(r e^{i \phi}\right)\right|}{V(r)} \longrightarrow h^{+}(\phi) \quad(r \notin \mathscr{E}, r \longrightarrow+\infty)
$$

uniformly in $\phi$; hence, by integration

$$
\lim _{\substack{r \rightarrow \infty \\ r \notin \mathscr{C}}} \frac{T(r, f)}{V(r)}=H_{+} \cdot
$$

In view of Lemma 2 , the restriction $r \notin \mathscr{E}$ may be omitted and (3.7) follows from (3.11).

By Jensen's formula and (1.5)

$$
\lim _{\substack{r \rightarrow \infty \\ r \notin \mathscr{E}}} \frac{N(r, 0)}{V(r)}=H
$$

once more Lemma 2 enables us to omit the restriction $r \notin \mathscr{E}$ because $N(r, o)$ is nondecreasing. Hence, in view of (3.7),

$$
\lim _{r \rightarrow \infty} \frac{N(r, o)}{T(r, f)}=\frac{H}{H_{+}},
$$

which implies (3.8). This completes the proof of Lemma 3 .

We conclude this section by noticing that, if $f(z)$ is of completely regular growth, (3.6) and (3.7) are valid and (3.1) holds (by (6) and one of the properties of proximate orders). Hence

$$
\lim _{r \rightarrow \infty} \frac{T(2 r, f)}{T(r, f)}=2^{\lambda} .
$$

This relation is needed in the proof of Theorem 2 .

4. Statement and proof of the main lemma. From Lemma B we now deduce

Lemma 4. Let $f(z)$ be entire and of finite order and let $h(\phi)$ be its Phragmén-Lindelöf indicator with respect to a Valiron growth function $V(r)$.

Assume that

$$
h(\phi) \equiv 0
$$

throughout the interval $[\alpha, \beta](0<\beta-\alpha<2 \pi)$.

Then, for

$$
\phi \in J=[\alpha+\eta, \beta-\eta]
$$

$$
\left(0<\eta<\frac{\beta-\alpha}{4}\right)
$$


(4.3) $\quad \log \left|f\left(r e^{i \phi}\right)-a\right| \leqq-K_{0} m\left(r, \frac{1}{f-a} ; J\right)+o(V(r)) \quad(r \rightarrow \infty)$, where a denotes any fixed, finite, complex quantity and

$$
K_{0}=4 \eta^{6} \pi^{-7}(\beta-\alpha)^{-1} \exp (-64 \pi /(\beta-\alpha))>0
$$

Moreover, if $f(z)$ is of completely regular growth with respect to $V(r)$ and if

$$
m\left(r, \frac{1}{f-a} ; J\right) \geqq \kappa_{0} T(r, f) \quad\left(r>r_{0}, 0<\kappa_{0}=\text { const. }\right)
$$

then

$$
\log \left|f\left(r e^{i \phi}\right)-a\right| \leqq-\frac{K_{0} \kappa_{0}}{2} T(r, f)
$$

Proof. Let $\epsilon>0$ be given. In view of a classical result [2; $p$. 46, Theorem 31], (4.1) yields

$$
\log \left|f\left(r e^{i \phi}\right)\right|<\frac{\epsilon}{2} V(r) \quad\left(r>r_{0}(\epsilon)\right),
$$

uniformly for all $\phi$ in $[\alpha, \beta]$.

Hence, by the elementary inequality

$$
\log \left|f\left(r e^{i \phi}\right)-a\right| \leqq \log ^{+}\left|f\left(r e^{i \phi}\right)\right|+\log ^{+}|a|+\log 2,
$$

we find, for $a$ fixed,

$$
\log \left|f\left(r e^{i \dot{\phi}}\right)-a\right|<\epsilon V(r) \quad\left(r>r_{0}(\epsilon)\right),
$$

uniformly for all $\phi$ in $[\alpha, \beta]$.

In order to apply Lemma B we first define the sector $S$ to be the set

$$
S: \frac{R}{\sigma} \leqq|z| \leqq \sigma R,\left|\arg z-\frac{\alpha+\beta}{2}\right| \leqq \frac{\beta-a}{2}=\gamma
$$

where

$$
\sigma=\exp \left(\frac{\pi(\beta-\alpha)}{4}\right)
$$

The parameter $\delta$ of Lemma B is defined by

$$
\delta=\frac{\eta}{\beta-\alpha} .
$$

The restrictions imposed by Lemma $\mathrm{B}$ on the quantities $\gamma, \sigma$ and 
$\delta$ are satisfied; moreover the choice of these quantities (and of $\eta$ ) is independent of $\epsilon$.

Now let

$$
z \in S, \quad \frac{R}{\sigma}>r_{0}(\epsilon)
$$

Then, by (4.7),

$$
\log |f(z)-a| \leqq \in V(\sigma R)<\epsilon\left(\sigma^{\lambda}+1\right) V(R),
$$

so that the function

$$
W(z)=(f(z)-a) \exp \left(-\epsilon\left(\sigma^{\lambda}+1\right) V(R)\right)
$$

is regular and its modulus does not exceed one on the set $S$. Hence the inequality (2.3) of Lemma B yields

$$
\log \left|W\left(R e^{i \dot{\phi}}\right)\right| \leqq-K m(R, 1 / W ; J),
$$

where $K$ may be chosen equal to

$$
K_{0}=64\left(\frac{\eta}{2 \pi}\right)^{6} \exp \left(-16 \frac{\pi^{2}}{\log \sigma}\right) / \log \sigma .
$$

In view of (4.8) this value of $K_{0}$ coincides with the one given in (4.4)

By (4.9)

$$
\log ^{+}\left|\frac{1}{W(z)}\right| \geqq \log ^{+} \frac{1}{|f(z)-a|}
$$

and therefore

$$
m\left(R, \frac{1}{W(z)} ; J\right) \geqq m\left(R, \frac{1}{f-a} ; J\right) \quad\left(R>\sigma r_{0}(\epsilon)\right) .
$$

Using (4.9) and (4.10) in (4.11), we find

$$
\begin{aligned}
\log \mid f\left(R e^{i \dot{\phi}}\right)- & a \mid \leqq-K_{0} m\left(R, \frac{1}{f-a} ; J\right) \\
+ & \in\left(\sigma^{\lambda}+1\right) V(R) \quad\left(R>\sigma r_{0}(\epsilon)\right) .
\end{aligned}
$$

Writing $r$ instead of $R$ and noticing that $\epsilon>0$ is arbitrary, we see that (4.12) implies (4.3).

Assume now that $f(z)$ is of completely regular growth with respect to $V(\mathrm{r})$. Then (3.6) and (3.7) are valid and we may replace, in (4.3), $o(V(r))$ by $o(T(r, f))$. Hence, in view of (4.5), we obtain (4.6). This completes the proof of Lemma 4 . 
5. Proof of Lemma 1. Consider the subset of $C$ defined by

$$
C_{+}=\{\phi: h(\phi)>0\} .
$$

Since $h(\phi)$ is continuous, $C_{+}$either coincides with $C$ or is the union of disjoint open intervals; moreover one of the properties of $h(\phi)$ [2; p. 47, Th. 32] asserts that each of these intervals has a length greater than or equal to $\min (\pi / \lambda, 2 \pi)$. Hence, if $p_{+}$denotes the number of components of $C_{+}$we have

$$
\begin{cases}p_{+} \leqq 2 \lambda & \left(\frac{1}{2} \leqq \lambda\right) \\ p_{+} \leqq 1 & \left(\lambda<\frac{1}{2}\right)\end{cases}
$$

and also, by (3.6),

$$
1 \leqq p_{+}
$$

It is now obvious that the set

$$
\widetilde{C}=C-C_{+}
$$

is the union of $\widetilde{p}\left(\leqq p_{+}\right)$closed intervals some of which may reduce to points. Let there be $p$ intervals of $\widetilde{C}$ which have a positive length; clearly

$$
p \leqq \widetilde{p} \leqq 2 \lambda
$$

We prove

LEMmA 5. Let $[\alpha, \beta](\alpha<\beta<\alpha+2 \pi)$ be any one of the $p$ nondegenerate intervals of $\widetilde{C}$. Then either (i) $h(\phi)<0$ throughout the interval $\alpha<\phi<\beta$ or (ii) $h(\phi) \equiv 0$ in $[\alpha, \beta]$.

Proof. Assume the lemma to be false. Then there must exist two interior points $\phi^{\prime}$ and $\phi^{\prime \prime}$ of $[\alpha, \beta]$ such that

$$
h\left(\phi^{\prime}\right)=0, \quad h\left(\phi^{\prime \prime}\right)<0 .
$$

This would imply the existence of three values $\phi_{1}, \phi_{2}, \phi_{3}$ such that

$$
\dot{\phi}_{1}<\phi_{2}<\dot{\phi}_{3}, \dot{\phi}_{3}-\dot{\phi}_{1}<\frac{\pi}{\lambda}
$$

and such that either

$$
h\left(\dot{\phi}_{1}\right) \leqq 0, h\left(\dot{\phi}_{2}\right)=0, h\left(\dot{\phi}_{3}\right)<0,
$$

or

$$
h\left(\dot{\phi}_{1}\right)<0, h\left(\dot{\phi}_{2}\right)=0, h\left(\dot{\phi}_{3}\right) \leqq 0 .
$$


Since each of these cases contradicts (1.2), Lemma 5 is proved.

By the assumptions of Lemma 1, there are exactly $q$ intervals of positive length on which $h(\phi) \equiv 0$ and, by Lemma 5 and (5.1),

$$
q \leqq p \leqq 2 \lambda \text {. }
$$

We thus obtain (1.8) and Lemma 1 is proved.

6. Proof of Theorem 2. Let $\left[\alpha_{j}, \beta_{j}\right](j=1,2,3, \cdots, q)$ be the $q$ intervals of positive length on which $h(\phi) \equiv 0$.

Consider the finite set $X$ formed by all the following points:

(i) the $2 q$ points $\alpha_{1}, \beta_{1}, \alpha_{2}, \beta_{2}, \cdots, \alpha_{q}, \beta_{q}$;

(ii) the points, if they exist, where $h(\phi)=0$ and $\phi \notin \bigcup_{j=1}^{q}\left[\alpha_{j}, \beta_{j}\right]$. Assume that $X$ has $P$ distinct elements; by (5.1), we clearly have

$$
P \leqq p+\widetilde{p} \leqq 4 \lambda \text {. }
$$

If $P=0$ then $h(\phi)>0$ for all $\phi$. This implies $q=0$ and, in view of the complete regularity of the growth of $f$, we also have $\delta(\tau, f)=0$ for all finite values of $\tau$. Theorem 2 is obvious in this case and, from now on we may assume $P \geqq 1$.

Let

$$
\theta_{1}, \theta_{2}, \cdots, \theta_{p} \quad\left(\theta_{1}<\theta_{2}<\cdots<\theta_{p}<\theta_{1}+2 \pi=\theta_{p+1}\right)
$$

be some circular arrangement of all the elements of $X$ and let

$$
0<\eta<\frac{1}{4} \min _{1 \leqq j \leqq p}\left\{\theta_{j+1}-\theta_{j}\right\} .
$$

Put

$$
\Lambda=\bigcup_{j=1}^{p}\left[\theta_{j}-\eta, \theta_{j}+\eta\right] .
$$

The set $C-\Lambda$ is the union of three disjoint subsets:

I. A "positive set" $0_{+}$on which $h(\phi)>0$.

II. A "negative set" $0_{-}$on which $h(\phi)<0$.

III. A "neutral set"

$$
\mathfrak{R}=\bigcup_{k=1}^{q}\left(\alpha_{k}+\eta, \beta_{k}-\eta\right) .
$$

Assume that there exist $q+1$ distinct values

$$
\tau_{1}, \tau_{2}, \cdots, \tau_{q+1},
$$

none of which is 0 or $\infty$, and such that 


$$
\delta\left(\tau_{j}, f\right)>0
$$$$
\left(j=1,2, \cdots, q_{+} 1\right) .
$$

Put

$$
3 \kappa=\min _{1 \leqq j \leqq q+1}\left\{\delta\left(\tau_{j}, f\right)\right\}
$$

and select $\eta$ in (6.2) so small that

$$
m\left(r, \frac{1}{f-\tau_{j}} ; \Lambda\right)<\kappa T(r, f) \quad\left(r>r_{0}, j=1,2, \cdots, q+1\right) .
$$

This is certainly possible because by Lemma A, Nevanlinna's first fundamental theorem, and (3.12), we find

$$
\begin{aligned}
m\left(r, \frac{1}{f-\tau_{j}} ; \Lambda\right) & \leqq 23 T(2 r, f) \mu\left(1+\log ^{+} \frac{1}{\mu}\right) \\
& \leqq 23\left(2^{\lambda}+1\right) T(r, f) \mu\left(1+\log ^{+} \frac{1}{\mu}\right) \quad\left(r>r_{0}\right),
\end{aligned}
$$

where

$$
\mu=\operatorname{meas} \Lambda=2 P \eta \text {. }
$$

The continuity of $h(\phi),(1.4)$ and (1.5) yield immediately

$$
\begin{aligned}
m\left(r, \frac{1}{f-\tau_{j}}\right. & \left.;\left\{0_{+} \cup 0_{-}\right\}\right)=0(1) \\
& (r \rightarrow+\infty, r \notin \mathscr{E}, j=1,2, \cdots, q+1) .
\end{aligned}
$$

The definition of $\delta(\tau, f),(6.5),(6.6)$ and (6.7) imply

$$
\begin{aligned}
m\left(r, \frac{1}{f-\tau_{j}} ; \kappa\right) & >\kappa T(r, f) \\
& \left(r>r_{0}, r \notin \mathscr{E}, j=1,2, \cdots, q+1\right) .
\end{aligned}
$$

Comparing (6.3) and (6.8) we see that, given an integer $j$, and an $r$, such that

$$
1 \leqq j \leqq q+1, r>r_{0}, r \notin \mathscr{E},
$$

we can find an integer $k=k(j, r)(1 \leqq k \leqq q)$ such that

$$
m\left(r, \frac{1}{f-\tau_{j}} ;\left[\alpha_{k}+\eta, \beta_{k}-\eta\right]\right)>\frac{\kappa}{q} T(r, f) .
$$

Hence, since there are $q+1$ acceptable values of $j$ and only $q$ acceptable values of $k$, it is possible to associate with each $r>r_{0}$, $r \notin \mathscr{E}$, two distinct values of $j$, say $j_{1}=j_{1}(r), j_{2}=j_{2}(r)$ such that

$$
k\left(j_{1}, r\right)=k\left(j_{2}, r\right)
$$


For a value of $k$ thus determined, we consider the sector

$$
S_{k}: \alpha_{k} \leqq \phi \leqq \beta_{k}, \quad r \geqq r_{0},
$$

and apply Lemma 4 twice: with

$$
\alpha=\alpha_{k}, \beta=\beta_{k}, \kappa_{0}=\frac{\kappa}{q}
$$

and first with $a=\tau_{j_{1}}$ then with $a=\tau_{j_{2}}$. Our choice of $\eta$, restricted by (6.1), certainly satisfies

$$
\eta<\frac{1}{4}\left(\beta_{k}-\alpha_{k}\right)
$$

In view of (6.9) and (6.10), the inequality (4.6) implies two distinct consequences:

$$
\log \left|f\left(r e^{i \phi}\right)-\tau_{j_{1}}\right| \leqq-\frac{K \kappa}{2 q} T(r, f) \quad\left(r>r_{0} ; r \notin \mathscr{E}\right),
$$

and

$$
\log \left|f\left(r e^{i \phi}\right)-\tau_{j_{2}}\right| \leqq-\frac{K \kappa}{2 q} T(r, f) \quad\left(r>r_{0} ; r \notin \mathscr{E}\right) .
$$

Both relations are valid for all $\phi$ in the interval $\left[\alpha_{k}+\eta, \beta_{k}-\eta\right]$ and therefore

$$
\begin{aligned}
\left|\tau_{j_{1}}-\tau_{j_{2}}\right| & \leqq\left|f\left(r e^{i \phi}\right)-\tau_{j_{1}}\right|+\left|f\left(r e^{i \phi}\right)-\tau_{j_{2}}\right| \\
& \leqq 2 \exp \left(-\frac{K \kappa}{2 q} T(r, f)\right) \quad\left(r>r_{0} ; r \notin \mathscr{E}\right) .
\end{aligned}
$$

Letting $r \rightarrow \infty \quad(r \notin \mathscr{E})$, we find

$$
\tau_{j_{1}}=\tau_{j_{2}} .
$$

This contradiction shows that the set (6.4) has too many elements. Hence the number of deficient values of $f(z)$, other than 0 and $\infty$, cannot exceed $q$. If $\delta(0, f)=0,(1.9)$ follows immediately from (1.8).

If $\delta(0, f)>0,(3.8)$ shows that we must have

$$
q<p
$$

Hence, by (5.1),

$$
q \leqq 2 \lambda-1
$$

and (1.9) follows again.

If $2 \lambda$ is a positive integer, we return to (5.1) and notice that 


$$
p_{+}=2 \lambda
$$

would imply $p=0$; therefore

$$
v(f)=1 \leqq 2 \lambda,
$$

and (1.10) follows in this case.

If (6.14) does not hold, then

$$
p \leqq p_{+} \leqq 2 \lambda-1
$$

and (1.10) follows immediately if $\delta(0, f)=0$. If $\delta(0, f)>0,(1.10)$ follows from (6.13) and (6.15).

This completes the proof of Theorem 2 .

\section{REFERENCES}

1. N. U. Arakeljan, Entire functions of finite order with an infinite set of deficient values, Dokl. Akad. Nauk SSSR 170 (1966), 999-1002.

2. M. L. Cartwright, Integral functions, Cambridge, 1956

3. A. Edrei and W. H. J. Fuchs, On meromorphic functions with regions free of poles and zeros, Acta Mathematica 108 (1962), 113-145.

4. A. Edrei and W. H. J. Fuchs, Bounds for the number of deficient values of certain classes of meromorphic functions, Proc. London Math. Soc. 12 (1962), 315-344.

5. B. Ja. Levin, Distribution of zeros of entire functions (English translation), New York, 1964.

6. R. Nevanlinna, Eindeutige Analytische Funktionen, second edition, Berlin, 1953.

7. A. Pfluger, Die Wertverteilung und das Verhalten von Betrag und Argument einer speziellen Klasse analytischer Funktionen I, Commentarii Math. Helv. 18 (1938), 180-214.

Received April 22, 1968. The research of the author was supported in part by a grant from the National Science Foundation GP-7407. It was done, under the guidance of Professor Edrei, in partial fulfillments of the $\mathrm{Ph}$. D. requirements at Syracuse University.

Syracuse University, Syracuse, New York, and

State University College at Buffalo, Buffalo, New York 



\section{Pacific Journal of Mathematics \\ Vol. 29, No. 1 \\ May, 1969}

Jorge Alvarez de Araya, A Radon-Nikodým theorem for vector and operator

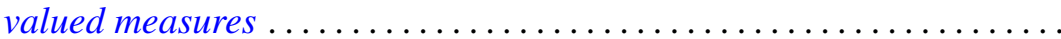

Deane Eugene Arganbright, The power-commutator structure of finite

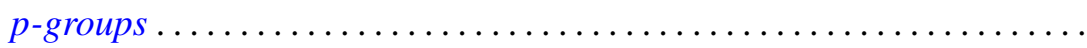

Richard Eugene Barlow, Albert W. Marshall and Frank Proschan, Some

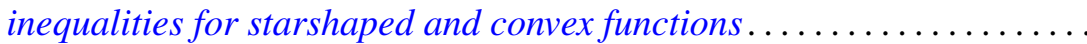

David Clarence Barnes, Some isoperimetric inequalities for the eigenvalues

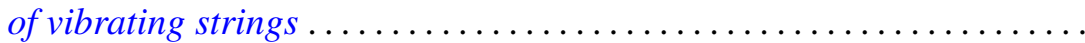

David Hilding Carlson, Critical points on rim-compact spaces ...........

Allan Matlock Weber Carstens, The lattice of pretopologies on an arbitrary

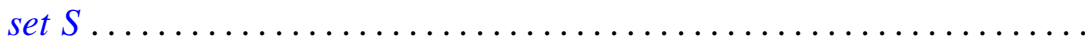

S. K. Chatterjea, A bilateral generating function for the ultraspherical

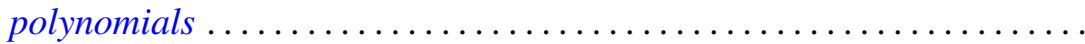

Ronald J. Ensey, Primary Abelian groups modulo finite groups ......... 77

Harley M. Flanders, Relations on minimal hypersurfaces ............ 83

Allen Roy Freedman, On asymptotic density in n-dimensions........... 95

Kent Ralph Fuller, On indecomposable injectives over artinian rings...... 115

George Isaac Glauberman, Normalizers of p-subgroups in finite groups . . . 137

William James Heinzer, On Krull overrings of an affine ring ........... 145

John McCormick Irwin and Takashi Ito, A quasi-decomposable abelian group without proper isomorphic quotient groups and proper isomorphic subgroups.

Allan Morton Krall, Boundary value problems with interior point boundary conditions

John S. Lowndes, Triple series equations involving Laguerre

polynomials

Philip Olin, Indefinability in the arithmetic isolic integers

Ki-Choul Oum, Bounds for the number of deficient values of entire functions whose zeros have angular densities..

R. D. Schafer, Standard algebras ....................

Wolfgang M. Schmidt, Irregularities of distribution. III.

Richard Alfred Tapia, An application of a Newton-like method to the Euler-Lagrange equation 